\title{
Dornase Alfa
}

National Cancer Institute

\section{Source}

National Cancer Institute. Dornase Alfa. NCI Thesaurus. Code C81664.

A recombinant human deoxyribonuclease I (rhDNAse) with selective DNA cleaving activity. Upon intrapleural administration, dornase alfa catalyzes the degradation of extracellular DNA in airway secretions, which can reduce their viscosity. Thus, dornase alfa may both promote the clearing of airway mucus and improve pulmonary function. 